\title{
Ein Feld in Bewegung: Friedens- und Konfliktforschung in herausfordernden Zeiten
}

\author{
Holger Niemann · Ursula Schröder
}

Online publiziert: 2. Mai 2020

(C) Der/die Autor(en) 2020

Zusammenfassung Wie kann die Friedens- und Konfliktforschung eine orientierende Funktion für den Umgang mit zunehmend komplexen und dynamischen Herausforderungen übernehmen? Der Wissenschaftsrat betont in seiner Evaluation des Felds, dass die Friedens- und Konfliktforschung wichtige Beiträge zur Bearbeitung aktueller gesellschaftlicher und politischer Herausforderungen leistet und der Bedarf an Expertise aus der Friedens- und Konfliktforschung zunehmen wird. Dieser Beitrag diskutiert aus der Perspektive der außeruniversitären Einrichtungen der Friedens- und Konfliktforschung, wie das Feld diese Funktion angesichts wachsender Herausforderungen ausüben kann. Hierzu beschreibt der Beitrag zunächst die Rolle außeruniversitärer Forschungseinrichtungen in einem komplementär strukturierten wissenschaftlichen Feld. Im zweiten Teil diskutieren wir, an welchen Stellen die Forschungsergebnisse der außeruniversitären Einrichtungen wichtige Impulse im Umgang mit neuen Herausforderungen setzen können. Wir argumentieren, dass es gilt, multiperspektivische Forschungszugänge zu stärken, Orientierungswissen für die gesellschaftliche und politische Praxis bereitzustellen und normative Positionen aufzuzeigen, um die orientierende Funktion der Friedens- und Konfliktforschung auch zukünftig zu erhalten und weiterzuentwickeln.

Schlüsselwörter Friedens- und Konfliktforschung · große gesellschaftliche Herausforderungen · Interdisziplinarität · Normativität · Orientierungswissen

H. Niemann $(\bowtie) \cdot$ U. Schröder $(\bowtie)$

Institut für Friedensforschung und Sicherheitspolitik (IFSH) / Universität Hamburg, Hamburg, Deutschland

E-Mail: niemann@ifsh.de; ursula.schroeder@uni-hamburg.de 


\section{A field in flux: peace and conflict studies for challenging times}

Abstract How can peace and conflict studies provide orientation for coping with increasingly complex and dynamic challenges? The evaluation report by the German Council of Science and Humanities (Wissenschaftsrat) emphasizes the importance of peace and conflict studies in targeting current societal and political challenges. The report also anticipates a growing demand for expertise from peace and conflict studies. The article discusses how peace and conflict studies can maintain a capacity for providing orientation in challenging times from the perspective of German peace research institutes. First, we examine the role of these specialized research institutes within the field of German peace and conflict studies. Second, we discuss how findings from these peace research institutes can make a difference in addressing novel challenges. We argue that for maintaining and enhancing the capacity of peace and conflict studies to provide orientation, it is necessary to strengthen a multiplicity of research perspectives, to make orientation knowledge available to politics and society, and to point out normative positions.

Keywords Peace and conflict studies · Grand societal challenges · Interdisciplinarity $\cdot$ Normativity $\cdot$ Orientation

\section{Einleitung}

Das Feld der Friedens-, Konflikt- und Sicherheitsforschung ist in Bewegung. Fundamentale Transformationen der globalen Sicherheitsarchitektur, das Ende etablierter internationaler Vertragswerke - insbesondere im Feld der Rüstungskontrolle und Abrüstung - und die Krise demokratischer Institutionen verbunden mit einem Erstarken populistischer Kräfte weltweit erfordern neue wissenschaftliche Analysen und Handlungsempfehlungen für die politische Praxis.

Der Wissenschaftsrat betont, dass die Friedens- und Konfliktforschung ,einen unverzichtbaren Beitrag zum Verständnis und zur Bearbeitung großer gesellschaftlicher Herausforderungen“" leistet (Wissenschaftsrat 2019, S. 8), der in Zukunft weiter zunehmen wird (Wissenschaftsrat 2019, S. 56). In unserem Artikel fragen wir, wie sich dieser Beitrag der Forschung in Zeiten des Wandels und des Umbruchs verändert. Große gesellschaftliche Herausforderungen formulieren hohe Erwartungen an die Wissenschaft und verlangen nach einer Neuformulierung ihrer Rolle in der Gesellschaft (Wissenschaftsrat 2015, S. 5). Sie erfordern die Zusammenführung unterschiedlicher Wissensbestände, um komplexe Fragen aus multiplen Perspektiven beleuchten zu können. Gleichzeitig stellen sie Herausforderungen für die gesellschaftliche und politische Praxis dar. Ihre Bearbeitung ist daher nicht nur eine Frage von Forschungszugängen und Methodologien, sondern auch von existierenden Ressourcen und Kapazitäten in einem vergleichsweise kleinen Forschungsfeld.

Wir zeigen in unserem Artikel zunächst, welchen Beitrag die außeruniversitären Forschungseinrichtungen der Friedens- und Konfliktforschung hier im Zusammenspiel mit anderen Akteuren des Felds leisten. So tragen die Arbeitsgemeinschaft für Friedens- und Konfliktforschung (siehe Engels und Schetter 2020) und die Deutsche 
Stiftung Friedensforschung (siehe Schneckener und Held 2020) über ihre Aktivitäten ganz zentral zur Institutionalisierung und Vernetzung des Felds bei. Die universitären Standorte vertreten das Feld wesentlich über ihre Aktivitäten in Lehre und Forschung, sind aber in höherem Maße durch die Prinzipien der wettbewerblichen Universität geprägt (siehe Bonacker 2020). Die außeruniversitären Institutionen haben in diesem komplementär gestalteten Feld durch ihre spezifischen Aktivitäten eine strukturbildende Rolle. Multiperspektivische und interdisziplinäre Herangehensweisen sind fest in den Strukturen der außeruniversitären Forschung etabliert und sie verfügen - auch aus historischen Gründen der Entwicklung des Felds - über eine besondere Expertise bei der Verbindung von Forschung mit Aktivitäten des Wissenstransfers und der Politikberatung, die die Bearbeitung zentraler Herausforderungen zukünftig verstärkt verlangen wird.

Zum anderen argumentieren wir, dass die Forschungsergebnisse außeruniversitärer Forschungseinrichtungen insbesondere in Zeiten einer wachsenden Komplexität vieler Herausforderungen eine orientierende Funktion haben. Wie Forschungseinrichtungen Orientierungswissen in diesen Zeiten bereitstellen können und welche Forschungszugänge hierzu sinnvoll sind, wird im zweiten Teil unseres Beitrags diskutiert. Wir argumentieren anhand von drei Fragen, dass es gilt, multiperspektivische Forschungszugänge zu stärken, Orientierungswissen für die gesellschaftliche und politische Praxis bereitzustellen und normative Positionen aufzuzeigen, um die orientierende Funktion der Friedens- und Konfliktforschung auch zukünftig zu erhalten.

\section{Der Beitrag der außeruniversitären Friedens- und Konfliktforschung}

Das Feld der Friedens- und Konfliktforschung steht nicht nur vor der Herausforderung, mit sehr begrenzten Ressourcen eine rapide steigende Zahl gesellschaftlich relevanter Themen und Fragestellungen zu bearbeiten. Vielmehr weisen diese eine neue Qualität auf und können als „große gesellschaftliche Herausforderungen“ verstanden werden. Der Wissenschaftsrat betont, dass große gesellschaftliche Herausforderungen sich z. B. durch inhaltliche Unschärfe, Vernetztheit und Zielpluralität auszeichnen (Wissenschaftsrat 2015, S. 16). Sie verlangen neue wissenschaftliche Herangehensweisen, da ihre Komplexität und Interdependenz ganzheitliche Ansätze benötigt. Gleichzeitig generieren sie auch politischen und gesellschaftlichen Handlungsdarf. Notwendig ist also auch ein Transfer wissenschaftlicher Expertise in politische und gesellschaftliche Entscheidungsprozesse.

Die Einrichtungen der außeruniversitären Forschung können einen wichtigen Beitrag zur Bearbeitung dieser Herausforderungen leisten, da sie sich in ihrer Struktur von der universitär verankerten Friedens- und Konfliktforschung unterscheiden. Während universitäre Forschung meist an Professuren innerhalb spezifischer Disziplinen gebunden ist, können außeruniversitäre Einrichtungen leichter Themen aufgreifen, die einen interdisziplinären Zuschnitt haben. Die außeruniversitären Einrichtungen verfügen zudem über eine lange Tradition, Forschung mit Wissenstransfer und Politikberatung zu verbinden. Zwar sind auch Universitäten zunehmend der third mission verpflichtet und verstehen den Austausch mit der Gesellschaft als 
wichtige Aufgabe. Letztlich bleibt eine starke Orientierung an gesellschaftlichen und politischen Anforderungen aber Kernaufgabe der außeruniversitären Einrichtungen. Auch der Wissenschaftsrat betont, dass insbesondere diese Einrichtungen hier eine „Bringschuld“ für das Feld erfüllen (Wissenschaftsrat 2019, S. 50).

Als spezialisierte Forschungseinrichtungen sind sie außerdem für die bundesweite und internationale Sichtbarkeit der Friedens- und Konfliktforschung relevant. Über ihre Forschungsprogramme verankern sie friedenswissenschaftliche Fragestellungen in regionalen und überregionalen Verbundforschungsprojekten. Als vergleichsweise große Institutionen bilden sie außerdem early career-Wissenschaftler*innen für das gesamte Forschungsfeld aus und wirken an der Koordination interdisziplinärer Studiengänge mit. Sie haben also eine besondere Funktion für die regionale Verdichtung und deutschlandweite Vernetzung des Felds. Die langjährige Rolle der außeruniversitären Institutionen für die öffentliche Sichtbarkeit des Felds zeigt sich auch in der Kontinuität spezifischer Publikationen, wie etwa dem standortübergreifenden jährlichen Friedensgutachten des Bonn International Center for Conversion (BICC), des Instituts für Entwicklung und Frieden (INEF), des Instituts für Friedensforschung und Sicherheitspolitik an der Universität Hamburg (IFSH) und des Leibniz-Instituts Hessische Stiftung Friedens- und Konfliktforschung (HSFK).

Die außeruniversitären Forschungseinrichtungen tragen zudem wichtige Bausteine zur inhaltlichen Agenda der Friedens- und Konfliktforschung bei. Bereits jetzt können einige vom Wissenschaftsrat als relevant eingestufte Themen insbesondere in den Einrichtungen der außeruniversitären Forschung abgebildet werden. Ein Beispiel ist das Feld der Rüstungskontrolle sowie der Analyse und Bewertung neuer Technologien, das traditionell sowohl in Deutschland als auch international eher in der außeruniversitären Forschungslandschaft bearbeitet wird. Am IFSH zum Beispiel arbeiten zurzeit 15 Wissenschaftler*innen in einem interdisziplinären Team an Fragen der Rüstungskontrolle und neuen Technologien. Die außeruniversitäre Forschung sollte in diesen Feldern auch in Zukunft aktiv involviert bleiben und ihr Engagement ausbauen, zeichnen sich aktuelle technologische Entwicklungen doch durch ihr hohes disruptives Potential für verschiedenste politische und gesellschaftliche Prozesse aus. Entwicklungen beispielsweise im Bereich der Informations- und Kommunikationstechnologien oder der künstlichen Intelligenz brauchen zukünftig verstärkt systematische und kritische wissenschaftliche Analysen ihrer möglichen friedenspolitischen Auswirkungen.

Ein zweites Beispiel ist das Feld der zivilen Konfliktbearbeitung und des gesellschaftlichen Zusammenlebens, das ebenfalls als Desiderat benannt wurde. Traditionell im Rahmen der Friedenspädagogik und Friedensbildung angesiedelt, waren solche Fragen der Konfliktbearbeitung und des inneren Friedens früher zentral für die deutsche Friedens- und Konfliktforschung. Aktuell verweist die verstärkte Beschäftigung mit Fragen innergesellschaftlicher Prozesse insbesondere - aber nicht nur - in den außeruniversitären Forschungsinstitutionen auf die neue Bedeutung, die diesem Feld zu Recht zugeschrieben wird. So untersucht das IFSH z. B. die Folgen von Radikalisierungsprozessen in sozialen Medien für den innergesellschaftlichen Frieden. Die außeruniversitäre Forschung verfügt über langjährige Erfahrung in der Untersuchung innergesellschaftlicher Prozesse und sollte auch zukünftig Mecha- 
nismen der friedlichen gesellschaftlichen Konfliktbearbeitung als festen Bestandteil ihrer Forschungsagenda berücksichtigen.

Daneben öffnet sich das Feld zunehmend für Forschung aus dem sicherheitspolitischen Bereich. Obwohl sicherheitspolitische Perspektiven für die außeruniversitäre Forschung immer schon relevant waren, wie es insbesondere die Geschichte des IFSH zeigt, werden die beiden Forschungsfelder häufig als Gegensätze verstanden, die in verschiedenen scientific communities mit unterschiedlichen methodischen und theoretischen Herangehensweisen untersucht werden. Dies ändert sich, seitdem verstärkt Fragen strategischer Orientierung oder auch Fragen aus dem Feld der critical security studies in der Friedens- und Konfliktforschung aufgenommen werden. Der Wissenschaftsrat begrüßt die Öffnung der Friedens- und Konfliktforschung gegenüber sicherheitspolitischen Fragen und betont, dass die spezifischen Zugänge der Friedens- und Konfliktforschung eine wichtige Ergänzung der existierenden Sicherheitsforschung darstellen können (Wissenschaftsrat 2019, S. 35).

\section{Wie kann die Friedens- und Konfliktforschung verstärkt multiperspektivisch forschen?}

Große gesellschaftliche Herausforderungen zeichnen sich als Querschnittsthemen durch grenzüberschreitende Interdependenzen und dynamische Entwicklungen aus (Wissenschaftsrat 2015, S. 16). Ihre Bearbeitung ist geprägt durch Disruptionen, unvollständige Informationen und Entscheidungsunsicherheiten. Für die Friedens- und Konfliktforschung bedeuten neue Phänomene wie der Klimawandel, die Digitalisierung, oder die globale Gesundheitspolitik daher nicht nur eine Ausweitung ihres Themenspektrums. Sondern sie verlangen auch verstärkt nach einer Zusammenführung unterschiedlicher Wissensbestände in multiperspektivischen Forschungszusammenhängen. Es ist daher folgerichtig, dass der Wissenschaftsrat der Friedensund Konfliktforschung eine engere Zusammenarbeit mit weiteren Disziplinen und Forschungsfeldern empfiehlt (Wissenschaftsrat 2019, S. 59).

Die außeruniversitären Einrichtungen stehen beispielhaft für die Integration unterschiedlicher wissenschaftlicher Disziplinen in längerfristige und größere Arbeitszusammenhänge in der Friedens- und Konfliktforschung. Dies gilt sowohl für die Zusammenarbeit zwischen Fächergruppen wie den Natur- und Sozialwissenschaften, aber auch für interdisziplinäre Herangehensweisen zwischen akademischen Disziplinen, etwa der Politikwissenschaft, Soziologie und Geographie. Derartige Formen interdisziplinärer Zusammenarbeit sind in den außeruniversitären Einrichtungen des Felds seit vielen Jahrzehnten gelebte Praxis. Die außeruniversitäre Friedens- und Konfliktforschung verfügt außerdem über Erfahrung bei der Formulierung Felderübergreifender Forschungsfragen. Ihre problemorientierte Herangehensweise ermöglicht es so, Forschungsthemen von Beginn an aus unterschiedlichen Perspektiven zu bearbeiten.

In den letzten Jahren haben insbesondere postkoloniale Ansätze (Dittmer 2018) und Beiträge zum local turn (Mac Ginty und Richmond 2013) die Notwendigkeit aufgezeigt, neben klassischen interdisziplinären Ansätzen verstärkt auch andere Wissensformen in der Friedens- und Konfliktforschung aufzugreifen. Die Berück- 
sichtigung alternativer Arten und Orte der Wissensproduktion ermöglicht es etwa indigenes oder traditionales Wissen stärker zu integrieren. In diesem Zusammenhang ist auch eine wachsende Sensibilität für die Grenzen des eigenen Wissens und der Positionalität von Forscherinnen und Forschern im Prozess der Wissensgenerierung betont worden, um lokale Effekte der Friedens- und Konfliktforschung angemessen zu bewerten zu können (Mac Ginty 2019, S. 271).

Aktuelle Diskussionen um co-creation von Wissen in der Interaktion mit gesellschaftlichen Akteuren und um neue Formen des Lernens, etwa in communities of practice oder Reallaboren, hinterfragen zudem die Trennung von Wissenschaft und Gesellschaft, Forscher*innen und Bürger*innen. Dieser Wandel der Forschungszugänge verweist auf Formen der Wissensproduktion, die häufig unter dem Begriff der Transdisziplinarität subsummiert werden. Sie basieren auf der Integration multipler Forschungsperspektiven und stellen die Unterscheidung von disziplinären und interdisziplinären Fragestellungen infrage. Damit ermöglichen sie eine problemorientierte Forschung, die nicht entlang fachlicher Grenzen, sondern gegenstandsbezogen strukturiert ist.

Solche Forschungszusammenhänge sind aber voraussetzungsvoll. Bereits interdisziplinäres Arbeit geht mit hohen Übersetzungskosten zwischen den Disziplinen einher (Brzoska 2012, S. 136). Die Integration weiterer (außerwissenschaftlicher) Akteur*innen intensiviert den Bedarf nach Verständigung noch. Zudem arbeiten Fördereinrichtungen häufig entlang fachdisziplinär organisierter Strukturen und Verfahren und erschweren damit die Finanzierung von Drittmittelprojekten, die den Querschnittscharakter großer gesellschaftlicher Herausforderungen in entsprechend multiperspektivische Herangehensweisen übersetzen.

Die Schwierigkeiten der Umsetzung inter- oder transdisziplinärer Arbeitsweisen stellt die grundsätzliche Notwendigkeit alternativer Forschungszugänge für die Bearbeitung großer gesellschaftlicher Herausforderungen jedoch nicht infrage. Vielmehr verweisen sie auf die Notwendigkeit, grundsätzlicher über Form und Funktion von Forschungszugängen und -prozessen sowie Wege der Generierung von Wissen zu reflektieren. Dabei geht es weniger um die Unvereinbarkeit disziplinärer und inter- bzw. transdisziplinärer Forschungsansätze, sondern vielmehr um pragmatische und sachlich begründbare Entscheidungen über spezifische Forschungsmodi. Dies ermöglicht es, die Vielfalt und die Kombinationsmöglichkeiten unterschiedlicher Organisationsformen von Wissenschaft in der Friedens- und Konfliktforschung problem- bzw. themenorientiert nutzen zu können (Bernshausen und Bonacker 2015, S. 250). Ein solcher Zugang über multiple Forschungsperspektiven bietet der Friedens- und Konfliktforschung die Möglichkeit, umfassende Orientierung im Umgang mit Querschnittsthemen bereit zu stellen. Eine kritische Reflexion verlangt aber auch, die Grenzen und Herausforderungen inter- und transdisziplinärer Forschungsmodi anzuerkennen und die Bedeutung disziplinärer Perspektiven nicht per se infrage zu stellen. 


\section{Wie kann die Friedens- und Konfliktforschung Orientierungswissen schaffen?}

Große gesellschaftliche Herausforderungen erzeugen aufgrund ihrer dynamischen Entwicklungen häufig Handlungsbedarfe in engen politischen Zeitfenstern (Wissenschaftsrat 2015, S. 16). Die Friedens- und Konfliktforschung kann orientierende Funktion übernehmen, da sie ,problemorientierte Grundlagenforschung“ (Wissenschaftsrat 2019, S. 36) betreibt und sich durch eine enge Verbindung zwischen Grundlagenforschung und Praxisorientierung auszeichnet. Obwohl beide Perspektiven im Feld sehr gut etabliert sind, existieren sie zumeist als voneinander getrennte Forschungszugänge (Wissenschaftsrat 2019, S. 36). Statt einer integrativen Herangehensweise existieren parallele Wege einerseits der Grundlagenforschung und anderseits anwendungsorientierter Forschung mit einem Fokus auf konkrete Problemlösung. Verantwortlich dafür sind vermutlich auch akademische Rahmenbedingungen und Förderstrukturen, die häufig eine klare Fokussierung auf einen dieser beiden Forschungszugänge verlangen. Eine Überwindung dieser Trennung wird vom Wissenschaftsrat entsprechend empfohlen (Wissenschaftsrat 2019, S. 56-57).

Die außeruniversitäre Friedens- und Konfliktforschung kann hier einen wichtigen Beitrag leisten. Ein enges Zusammenspiel zwischen Grundlagenforschung und Praxisorientierung ist insbesondere für die außeruniversitäre Friedens- und Konfliktforschung konstitutiv, da in diesen Institutionen der Wissenstransfer in Politik und Gesellschaft häufig Teil des satzungsgemäßen Auftrags ist. Neben den Ursprüngen des Felds in der Friedensbewegung (Krause 2019, S. 293) sind hierfür in Deutschland auch die Institutionalisierungsbestrebungen durch die Politik in den 1970er-Jahren verantwortlich (Lutz 2004, S. 29). Diese waren dezidiert mit der Erwartung verbunden, wissenschaftliche Erkenntnisse in einem zentralen gesellschaftlichen Handlungsfeld in praktische Politik übersetzen zu können. Durch die institutionell stärker verankerte Integration von Praxisorientierung und Grundlagenforschung kann die außeruniversitäre Friedens- und Konfliktforschung vermehrt problemorientiert forschen und übergreifendes Orientierungswissen bereitstellen.

Allerdings gilt es hierbei, Erwartungsmanagement zu betreiben. Von der Praxis werden häufig eindeutige Antworten verlangt, obwohl die Wissenschaft vor allem aus der Forschung ableitbare Fragen anbieten kann (Wulf 2011, S. 496). Gerade große gesellschaftliche Herausforderungen verlangen aber, die Grenzen des eigenen Wissens und seine beschränkte Umsetzung in konkrete Lösungsvorschläge anzuerkennen und offen zu thematisieren (Wissenschaftsrat 2015, S. 20). Die orientierende Funktion der Friedens- und Konfliktforschung zu stärken verweist daher nicht primär auf Forschung, die ausschließlich nachfrageorientiert wissenschaftlich abgesicherte Evidenz für politische Entscheidungen bereitstellt. Vielmehr geht es um die eigenständige und selbstbewusste Vermittlung komplexer Wissensbestände (Schröder 2019, S. 3). Denn nicht die Entwicklung vermeintlich einfacher Lösungsvorschläge für gesellschaftlich relevante Herausforderung, sondern eine Sensibilisierung für deren Komplexität und Vielfalt stärkt die Orientierungsfunktion der Friedens- und Konfliktforschung - und trägt damit auf anderem Wege zur Komplexitätsreduktion bei. Ziel muss es sein, wissenschaftliche Evidenz in ihren Entstehungskontexten einzubetten, durch alternative Deutungsmuster zu reflektieren und so gesellschaft- 
liche und politische Diskurse zu initiieren. Dies bietet die Möglichkeit, nicht nur Lösungen für erkannte Probleme zu generieren, sondern neue Begriffe und Lösungen bereitzustellen, um Probleme überhaupt erst zu erfassen und abzugrenzen, oder um ihre Dynamiken zu beschreiben und zu beobachten. Die Friedensforschung kann hier wesentlich dazu beitragen, nach neuen, vielleicht auch unorthodoxen, Lösungen zu suchen (Schröder 2019, S. 3).

Eine Fokussierung auf Orientierungswissen bietet zudem eine Alternative zur häufig stattfindenden Trennung zwischen Politikberatung und gesellschaftlichem Transfer. Der Wissenschaftsrat betont, dass die Nähe zur Politik ein „Charakteristikum“ der Friedens- und Konfliktforschung sei (Wissenschaftsrat 2019, S. 19). Und auch innerhalb des Felds wird Wissenstransfer häufig primär als Unterstützung politischer Entscheidungsprozesse durch wissenschaftliche Expertise verstanden (Nielebock 2017; Wulf 2011). Aber in Zeiten komplexer werdender Herausforderungen sollte Orientierungswissen mehr umfassen als die klassischen Formen des Wissenstransfers in die politische Exekutive. So müssen insbesondere diejenigen erreicht werden, deren Frieden und Sicherheit debattiert oder gar infrage gestellt wird, beispielsweise von gruppenbezogener Feindlichkeit bedrohte Personen, oder Gesellschaften mit eingeschränkter Resilienz im Umgang mit neuen Sicherheitsbedrohungen wie dem Klimawandel.

Es ist keine einfache Aufgabe, eine derartige Problemorientierung zu gewährleisten. Dies gilt erst recht, wenn damit nicht nur eine Kritik bestehender Verhältnisse auf abstrakter Ebene, sondern die ernsthafte Auseinandersetzung mit politischen und gesellschaftlichen Problemlagen gemeint ist (Krause 2019, S. 296). Es verlangt eine Auseinandersetzung mit vielfältigen und mitunter divergierenden Vorstellungen politischer und gesellschaftlicher Akteure. Notwendig ist auch eine kritische Beobachtung von Zuschreibungs- und Aneignungsprozessen von Kompetenzen und Verantwortungen. Aber angesichts der veränderten Bedingungen erscheint der Bedarf an Problemorientierung mehr denn je eine zentrale Aufgabe der Friedens- und Konfliktforschung zu sein. Orientierende Zugänge zu zentralen Fragen des Felds bieten zudem die Möglichkeit, die gesellschaftliche und politische Rolle der Friedensund Konfliktforschung zu stärken.

\section{Wie kann die Friedens- und Konfliktforschung in Zukunft normative Positionen vertreten?}

Mit der gesellschaftlichen und politischen Relevanz aktueller friedens- und sicherheitspolitischer Herausforderungen steigt auch der Bedarf nach normativer Orientierung. Der Wissenschaftsrat betont, dass gerade politische Entscheidungen über große gesellschaftliche Herausforderungen eine normative Reflexion durch die beteiligten Akteure erfordern (Wissenschaftsrat 2015, S. 20). Für ihre Bearbeitung ist also Wissen erforderlich, das im besten Falle auch normative Positionen für die Ausgestaltung konkreter gesellschaftlicher und politische Veränderungsprozesse aufzeigt.

Für die Friedens- und Konfliktforschung ist der Ruf nach normativen Positionen nicht neu. Vielmehr spielten solche Fragen über viele Jahrzehnte eine konstitutive 
Rolle für das Feld. Wie der Wissenschaftsrat hervorhebt, waren historisch insbesondere die Auseinandersetzungen zwischen ,kritischer“ und „traditioneller“ Friedensforschung maßgeblich für Diskussionen über die normativen Grundlagen des Felds (Wissenschaftsrat 2019, S. 21). Dieser Streit erklärt sich unter anderem aus unterschiedlichen theoretischen Zugriffen auf den Friedensbegriff (positiver vs. negativer Frieden) und aus dem charakteristischen Zusammenspiel zwischen Grundlagenforschung und Praxisorientierung. Diskussionen um die Normativität der Friedensforschung sind mittlerweile jedoch weniger polarisiert. So wird eher eine „Abkühlung“ (Bonacker 2011, S. 69) bzw. ,partielle Abstandsnahme“ (Brühl 2012, S. 176) von den ehemaligen Grabenkämpfen beobachtet, oder gar eine „Entnormativierung“ (Jaberg 2009, S. 39) des Felds konstatiert. Auch der Wissenschaftsrat kommt zu dem Schluss, dass der Streit um die normativen Positionen der Friedensforschung seit der Jahrtausendwende an Schärfe verloren hat (Wissenschaftsrat 2019, S. 22). Die veränderten wissenschaftlichen und gesellschaftlichen Rahmenbedingungen führen allerdings zu einem Bedeutungszuwachs normativer Orientierung. Die Friedens- und Konfliktforschung sollte sich daher verstärkt mit der Frage auseinandersetzen, wie sie im Rahmen ihrer Orientierungsfunktion auch zukünftig normative Positionen vermitteln kann.

Die außeruniversitären Forschungseinrichtungen können hier auf ihre über lange Zeit gewachsene Erfahrung aus dem Wissenstransfer und der Politikberatung zurückgreifen. Da diese Aktivitäten ganz wesentlicher Bestandteil des Arbeitsauftrags außeruniversitärer Institutionen sind, kann die Verständigung über zentrale normative Kriterien der eigenen Arbeit als Voraussetzung für den Erfolg ihrer Wissenstransferaktivitäten verstanden werden. Dabei ist in der außeruniversitären Forschung seit langem bekannt, dass insbesondere Aktivitäten der Politikberatung hierbei zu nichtintendierten Konsequenzen führen können. Denn der Transfer solchen Wissens in die Praxis erfolgt nicht linear, sondern ist Ergebnis politischer Selektionsprozesse (Schröder 2019, S. 2).

Die eigenen normativen Positionen zu reflektieren, ist daher auch als Frage der Forschungsethik zu verstehen. Gerade multiperspektivische Herangehensweisen hinterfragen die klassische Trennung zwischen wissenschaftlicher Expertise und dem Alltagswissen von Akteur*innen der Praxis. Dies kann zu Rollenkonflikten führen, etwa wenn außerwissenschaftliche Expertise nicht anerkannt wird, oder beispielsweise die Zusammenarbeit mit Bürger*innen in Austauschformaten zwischen Wissenschaft und Praxis durch epistemische Gewalt geprägt sind. Die eigenen normativen Positionen und damit verbundene Erwartungen, Motivationen und Ziele zu klären, kann daher als wichtiges Korrektiv in der Zusammenarbeit mit Praxispartner*innen verstanden werden. Normative Positionen aufzuzeigen ist somit nicht nur eine klassische Funktion des Felds, sondern auch als forschungsethische Selbstverortung und zur Reflexion des eigenen wissenschaftlichen Tuns und seiner Folgen bzw. Risiken notwendig.

Zudem steht insbesondere die außeruniversitäre Friedensforschung vor der Frage, wie normative Positionen auch gegen Widerstände aufrechterhalten werden können. Position zu beziehen kann herausfordernd sein, denn nicht immer stoßen die angebotenen Orientierungen auf Anklang oder Akzeptanz. Damit kann z. B. die Infragestellung von Finanzierungsmöglichkeiten oder Gesprächskanälen in Politik und 
Gesellschaft einhergehen. Angesichts steigender Abhängigkeiten von externen Finanzierungsmöglichkeiten besteht die Gefahr, dass Themen und Positionen allzu schnell externen Handlungszwängen unterworfen werden. Unter diesen schwierigen Bedingungen muss sichergestellt werden, dass Friedensforschung zugleich eine kritische Distanz behält und trotzdem konstruktive Beiträge zur politischen und gesellschaftlichen Praxis leisten kann.

Insgesamt ist die Frage also weniger $o b$, sondern vielmehr wie die Friedensforschung zukünftig normative Positionen vertreten möchte. Dabei kann es heute nicht mehr um alte Polarisierungen gehen, zu sehr haben sich die Forschungsgegenstände und -bedingungen geändert. Notwendig erscheint aber eine vertiefte Diskussion über die Möglichkeiten einer Friedens- und Konfliktforschung, die bereit ist Position zu beziehen und gleichzeitig Dogmatismus vermeiden kann. Dies erfordert Verständnis für die Positionalität des eigenen Wissens. Ebenso ist eine konsequente Einbeziehung internationaler Debatten erforderlich, um die Heterogenität und Diversität des Felds zu berücksichtigen (Mac Ginty 2019, S. 270-271). Denn die Vielfalt an Positionen aufzuzeigen und diese auch gegen Widerstände und dem Wunsch nach Eindeutigkeiten selbstbewusst zu vertreten, ist in Zeiten der Disruption etablierter Ordnungen nicht nur herausfordernd, sondern notwendig, will die Friedens- und Konfliktforschung ihre orientierende Funktion behalten.

\section{Schlussfolgerungen}

Die Friedens- und Konfliktforschung gibt es in Deutschland in der jetzigen Form seit etwas mehr als einem halben Jahrhundert. Sie hat in dieser Zeit globale Systemveränderungen analysiert, lokale Friedensprozesse begleitet und vor den Herausforderungen sicherheitspolitischer Risiken und Entscheidungen für den Frieden gewarnt. Der Bericht des Wissenschaftsrats hat die vielfältigen Leistungen der Friedens- und Konfliktforschung sowie die Relevanz des Felds für die wissenschaftliche und praktische Bearbeitung großer gesellschaftlicher Herausforderungen unterstrichen.

Strukturell konnte sich das Feld in den letzten Jahrzehnten gut etablieren. Die Friedens- und Konfliktforschung ist an den Universitäten und durch die außeruniversitären Forschungseinrichtungen fest verankert, eigene Studiengänge wurden erfolgreich aufgebaut. Zudem wurde die interne Vernetzung über Fachvereinigungen und einschlägige Publikationsformate gestärkt und das Feld hat sich insgesamt professionalisieren können. Die außeruniversitären Forschungseinrichtungen spielen dabei eine zentrale Rolle. Sie können im Zusammenspiel mit anderen Akteuren die Vielfalt und Breite des Felds repräsentieren und über ihre Forschungsagenden wesentlich zur thematischen Entwicklung des Fachs beitragen. Ihre umfangreiche Erfahrung in der Übersetzung wissenschaftlicher Erkenntnisse in Transferformate leistet dabei eine wichtige orientierende Funktion für Politik und Gesellschaft. In den aktuellen herausfordernden Zeiten wird diese Brückenfunktion zwischen Wissenschaft, Gesellschaft und Politik zunehmend wichtiger.

Die offensichtlich erfolgreiche Konsolidierung des Felds, die auch vom Wissenschaftsrat in seinem Bericht anerkannt wird, sollte aber kein Anlass für Stillstand sein. Vielmehr bietet die derzeitige Situation für das Feld eine wichtige Gelegen- 
heit, die Reflexion bisheriger Konsolidierungen mit der Identifikation zukünftiger Entwicklungspfade zu verbinden. Der Wissenschaftsrat macht dazu konkrete Vorschläge, mit denen sich die Friedens- und Konfliktforschung nun auseinandersetzen muss. Auch für die außeruniversitären Forschungseinrichtungen bietet sich damit eine wichtige Gelegenheit ihre Expertise in Forschung und Transfer auf neue Felder zu übertragen, alternative Formate des Dialogs mit Politik und Gesellschaft zu identifizieren und Kooperationen im Forschungsfeld zu intensivieren.

Neben die eigenständige Weiterentwicklung des Forschungsfelds durch die beteiligten Wissenschaftler*innen muss jedoch der wissenschaftspolitische Wille treten, die eingeleiteten Veränderungen auch budgetär abzusichern. Denn die Friedensund Konfliktforschung kann ,nicht alle aufgezeigten Herausforderungen aus eigener Kraft und mit den gegebenen Mitteln bewältigen“ (Wissenschaftsrat 2019, S. 56). Es bedarf also entsprechender wissenschaftspolitischer Entscheidungen, um die Friedens- und Konfliktforschung in ihrer Weiterentwicklung zu unterstützen und dabei die interne Vernetzung, die fachliche und methodische Vielfalt und ihre Internationalisierung zu fördern. Dies würde die wissenschaftliche Begleitung der großen Herausforderungen des Friedens auf gesellschaftlicher wie politischer Ebene durch die Friedens- und Konfliktforschung auch zukünftig ermöglichen.

Die Friedens- und Konfliktforschung ist in Bewegung. Dies ist kein Grund zur Sorge, hat das Feld doch bereits früher inhaltliche Kontroversen und politische Umbrüche überstanden. Sie muss und wird auch auf die fundamentalen Herausforderungen der gegenwärtig veränderten Rahmenbedingungen reagieren und ihre Erkenntnisse in die politische und gesellschaftliche Praxis vermitteln. Der Wissenschaftsrat hat durch seinen Bericht aufgezeigt, wo das Feld steht - und das gilt es zu begrüßen. Nun liegt es an den Beteiligten, die Weiterentwicklung der Friedensund Konfliktforschung aktiv zu gestalten.

Funding Open Access funding provided by Projekt DEAL.

Open Access Dieser Artikel wird unter der Creative Commons Namensnennung 4.0 International Lizenz veröffentlicht, welche die Nutzung, Vervielfältigung, Bearbeitung, Verbreitung und Wiedergabe in jeglichem Medium und Format erlaubt, sofern Sie den/die ursprünglichen Autor(en) und die Quelle ordnungsgemäß nennen, einen Link zur Creative Commons Lizenz beifügen und angeben, ob Änderungen vorgenommen wurden.

Die in diesem Artikel enthaltenen Bilder und sonstiges Drittmaterial unterliegen ebenfalls der genannten Creative Commons Lizenz, sofern sich aus der Abbildungslegende nichts anderes ergibt. Sofern das betreffende Material nicht unter der genannten Creative Commons Lizenz steht und die betreffende Handlung nicht nach gesetzlichen Vorschriften erlaubt ist, ist für die oben aufgeführten Weiterverwendungen des Materials die Einwilligung des jeweiligen Rechteinhabers einzuholen.

Weitere Details zur Lizenz entnehmen Sie bitte der Lizenzinformation auf http://creativecommons.org/ licenses/by/4.0/deed.de.

\section{Literatur}

Bernshausen, Sirin, und Thorsten Bonacker. 2015. Ist die Friedens- und Konfliktforschung auf dem Weg zur Disziplin? Ein wissenschaftssoziologischer Blick auf die Bedeutung von Studiengängen für die Ausdifferenzierung von Disziplinen. In Konflikte vermitteln? Lehren und Lernen in der Friedens- und Konfliktforschung, Hrsg. Mathias Bös, Lars Schmitt, und Kerstin Zimmer, 247-268. Wiesbaden: VS. 
Bonacker, Thorsten. 2011. Forschung für oder Forschung über den Frieden? Zum Selbstverständnis der Friedens- und Konfliktforschung. In Friedens- und Konfliktforschung, Hrsg. Peter Schlotter, Simone Wisotzki, 46-78. Baden-Baden: Nomos.

Bonacker, Thorsten. 2020. Die Grenzen des Wettbewerbs. Zur Evaluation der Friedens- und Konfliktforschung (nicht nur) aus universitärer Sicht. Zeitschrift für Friedens- und Konfliktforschung. https://doi. org/10.1007/s42597-020-00028-y.

Brühl, Tanja. 2012. Friedensforschung als „Superwissenschaft“ oder Sub-Disziplin? Zum Verhältnis der Friedens- und Konfliktforschung und der Internationalen Beziehungen. Zeitschrift für Internationale Beziehungen 19(1):171-183.

Brzoska, Michael. 2012. Friedensforschung und Internationale Beziehungen - Lob der Verschiedenheit. Zeitschrift für Internationale Beziehungen 19(1):127-141.

Dittmer, Cordula (Hrsg.). 2018. Dekoloniale und postkoloniale Perspektiven in der Friedens- und Konfliktforschung. Verortungen in einem ambivalenten Diskursraum. ZeFKo Sonderband, Bd. 2. BadenBaden: Nomos.

Engels, Bettina, und Conrad Schetter. 2020. Die Gelegenheit nutzen: Die Evaluation der Friedens- und Konfliktforschung durch den Wissenschaftsrat aus der Perspektive der Arbeitsgemeinschaft für Friedens- und Konfliktforschung (AFK). Zeitschrift für Friedens- und Konfliktforschung. https://doi.org/ 10.1007/s42597-020-00027-z.

Ginty, Mac Roger. 2019. Complementarity and interdisciplinarity in peace and conflict studies. Journal of Global Security Studies 4(2):267-272.

Ginty, Mac Roger, und Oliver Richmond. 2013. The local turn in peacebuilding: A critical agenda for peace. Third World Quarterly 34(5):763-783.

Jaberg, Sabine. 2009. Vom Unbehagen am Normverlust zum Unbehagen mit der Norm? Zu einem fundamentalen Problem der neueren Friedensforschung. Hamburger Beiträge zur Friedensforschung und Sicherheitspolitik, Bd. 152. Hamburg: Institut für Friedensforschung und Sicherheitspolitik an der Universität Hamburg.

Krause, Keith. 2019. Emancipation and critique in peace and conflict research. Journal of Global Security Studies 4(2):292-298.

Lutz, Dieter S. 2004. Friedensforschung - normativ, interdisziplinär, praxisorientiert. In Friedens- und Konfliktforschung in Deutschland, Hrsg. Ulrich Eckern, Leonie Herwartz-Emden, und Rainer-Olaf Schultze, 23-32. Wiesbaden: VS.

Nielebock, Thomas. 2017. Ethische Herausforderungen friedenswissenschaftlicher Politikberatung. In Handbuch Friedensethik, Hrsg. Ines-Jacqueline Werkner, Klaus Ebeling, 927-940. Wiesbaden: VS.

Schneckener, Ulrich, und Thomas Held. 2020. Impulsgeberin und Anstifterin: Die Deutsche Stiftung Friedensforschung im Lichte der Evaluation. Zeitschrift für Friedens- und Konfliktforschung. https://doi. org/10.1007/s42597-020-00029-x.

Schröder, Ursula. 2019. Orientierungswissen durch Friedens- und Sicherheitsforschung. IFSH Policy Brief 01/19. Hamburg: Institut für Friedensforschung und Sicherheitspolitik an der Universität Hamburg.

Wissenschaftsrat. 2015. Zum wissenschaftspolitischen Diskurs über große gesellschaftliche Herausforderungen. Positionspapier. Drs. 4594-15. Stuttgart: Wissenschaftsrat.

Wissenschaftsrat. 2019. Empfehlungen zur Weiterentwicklung der Friedens- und Konfliktforschung. Drs. 7827-19. Gießen: Wissenschaftsrat.

Wulf, Herbert. 2011. Friedensforschung und Politikberatung. In Handbuch Frieden, Hrsg. Hans-Jürgen Gießmann, Bernhard Rinke, 495-502. Wiesbaden: VS. 